\title{
Multichannel Wavelength Conversion of 40Gbit/s NRZ DPSK Signals in a Highly Nonlinear Dispersion Flattened Lead Silicate Fibre
}

\author{
Angela Camerlingo, Francesca Parmigiani, Xian Feng, Francesco Poletti, Peter Horak, Wei H. \\ Loh, Periklis Petropoulos and David J. Richardson \\ Optoelectronics Research Centre, University of Southampton, Southampton SO17 1BJ, United Kingdom \\ Email: anc@orc.soton.ac.uk
}

\begin{abstract}
We experimentally demonstrate the wavelength conversion of three wavelength multiplexed $40 \mathrm{Gbit} / \mathrm{s}$ Differential Phase Shift Keyed (DPSK) signals in a $2.2 \mathrm{~m}$ length of highly nonlinear, dispersion tailored W-type lead-silicate optical fibre.

OCIS codes: (060.2280) Fiber design and fabrication; (060.4370) Nonlinear optics, fibers; (190.4380)

Nonlinear optics, four-wave mixing;
\end{abstract}

\section{Introduction}

Wavelength conversion is a crucial operation in high speed wavelength division multiplexed (WDM) optical networks for the implementation of essential applications, such as routing or switching. Efficient wavelength conversion of signals can be realised by exploiting nonlinear processes in a medium and amongst them four-wave-mixing (FWM) is regarded as the most promising, especially because of its modulation format-agnostic nature. The most important fibre parameters for achieving efficient FWM are a high effective nonlinear coefficient, a low and flat dispersion profile and a short length. Highly nonlinear soft glass fibres have already proven to be highly suitable candidates to accomplish this task [1-3], offering the possibility to combine a high effective nonlinearity coefficient with a tailored dispersion profile. Here we consider a small-core W-type soft glass fibre comprising three commercial (Schott) lead-silicate glasses that has been designed to have low dispersion in the $1.55 \mathrm{~m}$ band. We demonstrate simultaneous FWM-based wavelength conversion of three WDM $40 \mathrm{Gbit} / \mathrm{s}$ non-return to zero (NRZ) differential phase shift keyed (DPSK) signals in a $2.2 \mathrm{~m}$ length of this fibre.

\section{Experimental setup and results}

The experimental setup for the FWM-based wavelength converter is shown in Fig. 1. The nonlinear medium in the system was a lead-silicate W-type fibre, an SEM of which is also shown in the figure. The fibre core (1.6 m in diameter) was made of a high index glass, Schott SF57 (n=1.82 @ $1550 \mathrm{~nm})$ which was surrounded by a first cladding ( $6.7 \mathrm{~m}$ in diameter) made of a low index glass, Schott LLF1

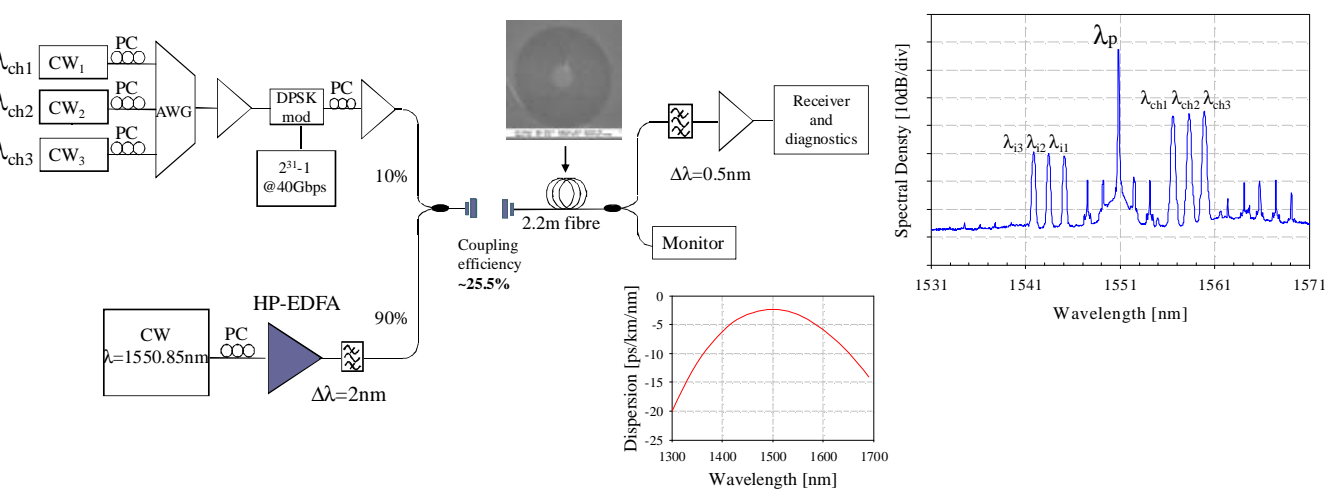

Fig.1: Experimental setup of the FWM-based wavelength converter. Insets: SEM image of the W-type fibre; numerical simulation of the fibre dispersion as a function of wavelength; optical spectrum obtained at the output of the W-type fibre.

(n=1.53 @ $1550 \mathrm{~nm}$ ). The outer cladding was made of a different glass with a higher refractive index, Schott SF6, (n=1.76 @ 1550nm). The insertion of the ring of LLF1 glass allowed manipulation of the fibre dispersion, whereas the inclusion of the outer cladding ensured single mode operation at telecoms wavelengths. This fibre design was the same as the one reported in [4]; however optimisation in the fabrication procedures has allowed a significant decrease in the fibre propagation losses from $4.8 \mathrm{~dB} / \mathrm{m}$ 
to $2.1 \pm 0.2 \mathrm{~dB} / \mathrm{m}$ in this instance, as measured using the cut-back method. By measuring the self-phasemodulation (SPM) response of the fibre, the nonlinear coefficient was found to be $\sim 820 \mathrm{~W}^{-1} \mathrm{~km}^{-1}$. The dispersion of the fibre was evaluated to be $\sim-3 \mathrm{ps} / \mathrm{nm} / \mathrm{km}$ at $1.55 \mathrm{~m}$ through FWM measurements. The simulated dispersion profile of the fibre is shown in the inset to Fig.1.

The outputs of three continuous wave (CW) lasers ( ${ }_{\mathrm{ch} 1}=1556.86 \mathrm{~nm}, \quad{ }_{\mathrm{ch} 2}=1558.58 \mathrm{~nm}$, ${ }_{\mathrm{ch} 3}=1560.20 \mathrm{~nm}$ ) were multiplexed together using an arrayed waveguide grating (AWG) and modulated with a $2^{31}-1$ pseudorandom bit sequence (PRBS) at 40Gbit/s. The pump signal for the FWM process was generated by a CW laser $(\mathrm{p}=1550.85 \mathrm{~nm})$ and amplified using a high power erbium-doped fibre amplifier (HP-EDFA). A bandpass filter (BPF) was employed at the output of the high power EDFA to reject any undesired out-of-band amplified spontaneous emission (ASE) noise. In order to control the relative power levels of the signals and the pump and to avoid any nonlinear interaction in the EDFA, the three data channels were amplified separately from the pump using a second EDFA. The states of polarisation of the two beams were controlled by two polarization controllers (PCs) and were aligned to a common polarization axis of the fibre. The $\mathrm{CW}$ pump and the data channels were then combined in a 90/10 coupler and free-space launched into the fibre. The coupling efficiency was estimated to be $\sim 25.5 \%$ and the average powers of the CW pump and the three DPSK signals at the very input of the fibre were $23 \mathrm{dBm}$ and $8.7 \mathrm{dBm}$, respectively.

The inset to Fig. 1 shows the optical spectra recorded after the W-type fibre. The idlers exhibited an optical signal to noise ratio (OSNR) greater than $25 \mathrm{~dB}$ and a uniform conversion efficiency (defined as the ratio of the idler power to the corresponding channel power) of $-12 \mathrm{~dB}$. The output spectrum indicates the existence of some low-power spurious frequency components, which were mixing products between the various WDM channels and the pump. These components were clearly at different wavelengths to the WDM channels, or their idlers, and did not affect the performance of the system. Additional measurements on the converted idlers also showed negligible nonlinear cross-talk. Each of the idlers was carefully filtered and characterised in terms of eye diagrams and bit error ratio (BER) measurements. Fig. 2 (left) shows a comparison between the DPSK demodulated eye diagrams of each channel at the transmitter output with that of its corresponding idler. The good quality of the conversion process was reflected in the BER measurements (Fig. 2 (right)), which showed a power penalty between $0.5 \mathrm{~dB}$ and $2 \mathrm{~dB}$ for the three converted channels.

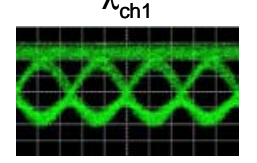

ch2

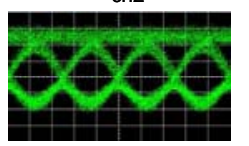

ch3

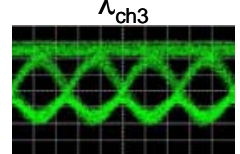

Fig 2 (Left): Eye diagrams of each channel and of the corresponding filtered idlers. (Right): BER of each channel and of the corresponding filtered idler.

\section{Conclusions}

We have experimentally demonstrated a FWM-based multichannel conversion scheme using three DPSK channels at 40Gbit/s in a $2.2 \mathrm{~m}$ length of highly nonlinear, dispersion flattened soft glass fibre. This work was partially funded by the European Union STREP project PHASORS (FP7-ICT-2007-2 22457).

\section{References}

[1] S. Asimakis et al., "Towards efficient and broadband four-wave-mixing using short-length dispersion tailored lead silicate holey fibres", Opt. Exp., vol. 15, no. 2, pp 596-601, 2007.

[2] K. K. Chow et al., "Four-wave-mixing based widely tunable wavelength conversion using 1-m dispersion-shifted bismuthoxide photonic crystal fibre", Opt. Exp., vol. 15, no. 23, pp 15418-15423, 2007.

[3] A. Camerlingo et al., "Four-wave mixing-based wavelength conversion in a Short-Length of a Solid 1D Microstructured Fibre, ECOC 2009, Vienna.

[4] A. Camerlingo et al., "Wavelength Conversion in a Short Length of a Solid Lead-Silicate Fibre", IEEE Photon. Technology Lett, vol. 22, no. 9, pp. 628-630, 2010. 